\title{
FRAÇÕES NITROGENADAS E EFICIÊNCIA NUTRICIONAL EM LINHAGENS DE FEIJOEIRO (Phaseolus vulgaris L.)
}

\author{
Nitrogen fractions and nutritional efficiency in bean lineages (Phaseolus vulgaris L.)
}

\author{
Fabiano José do Lago ${ }^{1}$, Antonio Eduardo Furtini Neto², Isabela Volpi Furtini, \\ Magno Antonio Patto Ramalho ${ }^{4}$, Ivana de Marco Fonseca Horta ${ }^{5}$
}

\begin{abstract}
RESUMO
Visando a estudar a associação das frações nitrogenadas nas folhas e a eficiência no uso de nitrogênio (EUN) em feijoeiro (Phaseolus vulgaris L.), foram avaliadas 12 linhagens na presença e ausência do nutriente, em três locais no sul de Minas Gerais. Em cada local, experimentos distintos, com e sem aplicação de nitrogênio, foram instalados no delineamento em blocos casualizados (DBC), com três repetições. As parcelas foram constituídas por uma linha de três metros de comprimento. Avaliou-se a produtividade de grãos $\left(\mathrm{kg} \mathrm{ha}^{-1}\right)$, os teores de $\mathrm{N}$-total nas folhas e nos grãos, e as frações do $\mathrm{N}$-total nas folhas $\left(\mathrm{N}\right.$-solúvel, $\mathrm{N}-\mathrm{NO}_{3}{ }^{-} \mathrm{N}$-insolúvel e $\mathrm{N}$ orgânico solúvel). A produtividade média de grãos com a aplicação de nitrogênio foi $63,7 \%$ superior à obtida sem o nutriente. As linhagens ESAL 629 e MA-I-2.5 apresentaram as maiores produtividades de grãos em todos os locais e níveis de N, e também foram mais eficientes em relação à EUN. Os teores de nitrato foram maiores sem a aplicação de N, e, por outro lado, as demais frações de $\mathrm{N}$ e do N-total na folhas e nos grãos foram mais elevadas quando houve o fornecimento de nitrogênio. Não se constatou boa associação entre os teores de $\mathrm{N}$-total e as frações de $\mathrm{N}$ nas folhas com a EUN entre as linhagens estudadas; contudo, nas linhagens ESAL 629 e MA-I-2.5 houve evidências de que as frações de $\mathrm{N}$ e N-total nas folhas poderiam explicar a sua maior EUN.
\end{abstract}

Termos para indexação: nitrogênio, metabolismo mineral, feijoeiro, Phaseolus vulgaris

\begin{abstract}
Twelve bean lineages (Phaseolus vulgaris L.) in the presence and absence of nitrogen in three places in the south of Minas Gerais were evaluated aiming to study the relationship between nitrogen fractions in leaves and the efficiency of nitrogen usage in the bean crop. In each place, distinct experiments with and without nitrogen application were installed using randomized blocks design, with three replications. The parcels were constituted by three-meter-long lines. Grain productivity $\left(\mathrm{kg} \mathrm{ha}^{-1}\right)$, amounts of total $\mathrm{N}$ in leaves and grains, and total $\mathrm{N}$ in leaf fractions (soluble- $\mathrm{N}, \mathrm{NO}_{3}^{-}-\mathrm{N}$, insoluble- $\mathrm{N}$, and soluble organic- $\mathrm{N}$ ) were evaluated. The average of grain productivity with nitrogen application was $63.7 \%$ higher than the productivity without this nutrient. The ESAL 629 and MA-I-2.5 presented higher grain productivities in all places and in all $\mathrm{N}$ levels and they were also more efficient in relation to $\mathrm{N}$ usage. The nitrate levels had higher values without $\mathrm{N}$ application than with it, and, on the other hand, the other fractions of $\mathrm{N}$ and total- $\mathrm{N}$ in leaves and grains presented higher values when there was nitrogen addition instead of absence of it. There was no good relationship between amounts of total-N and $\mathrm{N}$ fractions in leaves, and efficiency of $\mathrm{N}$ usage among the studied lineages; however, in the case of ESAL 629 and MA-I-2.5 there were evidences that the $\mathrm{N}$ fractions and total- $\mathrm{N}$ in leaves could explain their higher efficiency of $\mathrm{N}$ usage.
\end{abstract}

Index terms: Nitrogen, mineral metabolism, bean crop, Phaseolus vulgaris.

(Recebido em 25 de maio de 2007 e aprovado em 14 de dezembro de 2007)

\section{INTRODUÇÃO}

A cultura do feijoeiro (Phaseolus vulgaris L.) ainda apresenta baixos níveis de produtividade, ocasionados por vários fatores, dentre os quais destacam-se a cultivar utilizada, o manejo da cultura, e os estresses bióticos e abióticos.

Entre os estresses abióticos a que o feijoeiro é submetido, aqueles relacionados à falta de nutrientes do solo são os mais expressivos. No caso do nitrogênio (N), nutriente mais extraído e exportado pelo feijoeiro (VIEIRA, 2006), tem-se uma situação especial, porque embora o feijoeiro apresente a fixação simbiótica desse nutriente, este processo é insuficiente para suprir a demanda da planta pelo mesmo (ALVES, 2002; SILVA, 2002). Por essa razão, na maioria das vezes, é imprescindível sua aplicação na

\footnotetext{
'Engenheiro Agrônomo, Mestre em Solos e Nutrição de Plantas - Plantar S/A Reflorestamento - Rua Doutor Veloso, 980 - Centro - $39400-970$ - Montes Claros, - fabiano-lago@plantar.com.br

'Engenheiro Agrônomo, Doutor em Solos e Nutrição de Plantas, Professor - Departamento de Ciência do Solo/DCS - Universidade Federal de Lavras/ UFLA - Cx. P. 3037 - 37200-000 - Lavras, MG - afurtini@ufla.br

${ }^{3}$ Engenheira Agrônoma, Mestranda em Genética e Melhoramento de Plantas - Departamento de Biologia/DBI - Universidade Federal de Lavras/UFLA - Cx. P. 3037 - 37200-000 - Lavras, MG - isafurtini@yahoo.com.br - Bolsista CNPq

${ }^{4}$ Engenheiro Agrônomo, Doutor em Genética e Melhoramento de Plantas, Professor - Departamento de Biologia/DBI - Universidade Federal de Lavras/ UFLA - Cx. P. 3037 - 37200-000 - Lavras, MG - agnoapr@ufla.br

${ }^{5}$ Geógrafa, Mestre em Engenharia Florestal - Departamento de Engenharia Florestal/DCF - Universidade Federal de Lavras/UFLA - Cx. P. 3037 37200-000 - Lavras, MG - idmfh@hotmail.com
} 
cultura, para que a produção não seja afetada negativamente. A maximização do uso de $\mathrm{N}$ pelo feijoeiro é de grande importância, tanto em função de aspectos econômicos, quanto ambientais, uma vez que este nutriente apresenta riscos ao meio ambiente por ser potencialmente contaminante de lençóis freáticos e pelo fato de que os fertilizantes nitrogenados são caros e produzidos a partir de fontes não renováveis.

Há vários relatos na literatura de diferenças entre linhagens e, ou cultivares de várias espécies quanto à tolerância a elementos tóxicos, bem como ao uso de nutrientes dos solos (BRASIL, et al., 2007). Com relação ao nitrogênio no feijoeiro, as pesquisas são mais escassas. De maneira geral, tem-se obtido respostas da cultura à adubação nitrogenada; entretanto, a freqüência e a amplitude da resposta variam de região para região e, ainda, dentro da mesma região, em função do clima e das condições fitossanitárias (FRANCO, 1997; VIEIRA, 1998).

Furtini et al. (2006) avaliando 100 linhagens de feijoeiro no sul de Minas Gerais na presença e ausência de $\mathrm{N}$ em cobertura, observaram diferenças entre as linhagens com relação à eficiência no uso do nutriente, embora não tenham até o momento nenhuma informação a respeito da estratégia que proporciona à planta uma maior ou menor eficiência. Especula-se que uma alternativa seria o estudo das frações de nitrogênio nas folhas, as quais poderiam auxiliar na seleção de plantas e, ou linhagens com maior eficiência no uso do N.

Neste trabalho, objetivou-se avaliar a associação das frações nitrogenadas nas folhas com a eficiência no uso de nitrogênio em linhagens de feijoeiro.

\section{MATERIAL E MÉTODOS}

Experimentos distintos foram conduzidos durante a safra da "seca", fevereiro a maio de 2005, em três municípios no sul de Minas Gerais: Lavras ( $21^{\circ} 14^{`} \mathrm{~S}, 44^{\circ}$ $59^{\circ}$ W e a uma altitude média de $919 \mathrm{~m}$ ) em um Latossolo Vermelho distroférrico (LVdf); Ijaci $\left(21^{\circ} 10^{`} \mathrm{~S}, 44^{\circ} 55^{`} \mathrm{~W}\right.$ e a uma altitude média de $805 \mathrm{~m}$ ) em um Argissolo Vermelho Amarelo (PVA); e Lambari ( $21^{\circ} 50^{`} \mathrm{~S}, 44^{\circ} 21^{`} \mathrm{~W}$ e a uma altitude média de $887 \mathrm{~m}$ ) em Neossolo Flúvico (RU). Os atributos dos solos onde foram conduzidos os experimentos são apresentados na Tabela 1.

Foram utilizadas seis linhagens com maiores índices de eficiência de uso de nitrogênio (EUN), (RC-I-3, ANLAV-51, CV-46, CNFC 8063, MA-I-6.10, ESAL 629), e seis com menores índices (CIII-R-3-19, CNFC 8060, CI-107, OPS-30, H-9, MA-I-2.5), de acordo com Furtini et al. (2006), selecionadas entre 100 linhagens avaliadas em experimentos anteriores. A eficiência do uso de nitrogênio foi avaliada pela expressão proposta por Thung (1990):

$$
\mathrm{EUN}=\left(\mathrm{P}_{\mathrm{c} / \mathrm{N}}-\mathrm{P}_{\mathrm{s} / \mathrm{N}}\right) / \mathrm{D}_{\mathrm{dN}}
$$

em que:

$\mathrm{P}_{\mathrm{c} / \mathrm{N}}$ : produtividade de grãos das linhagens no maior nível de N;

$\mathrm{P}_{\mathrm{s} / \mathrm{N}}$ : produtividade de grãos das linhagens no menor nível de $\mathrm{N}$;

$\mathrm{D}_{\mathrm{dN}}$ : diferença entre o maior e o menor nível de $\mathrm{N}$.

Em todos os locais foram conduzidos dois experimentos distintos e contíguos, com os mesmos tratos

Tabela 1 - Atributos dos solos à profundidade de 0 a 20 cm nas áreas experimentais em Lavras, Lambari e Ijaci.

\begin{tabular}{lccc}
\hline \multirow{2}{*}{ Análise química } & \multicolumn{2}{c}{ Locais } & Ijaci \\
\cline { 2 - 4 } & Lavras & Lambari & 5,0 \\
\hline $\mathrm{pH} \mathrm{em} \mathrm{H} \mathrm{H}_{2} \mathrm{O}$ & 5,2 & 5,1 & 9,5 \\
$\mathrm{P}\left(\mathrm{mg} \mathrm{dm}^{-3}\right)$ & 42,4 & 32,9 & 35,0 \\
$\mathrm{~K}\left(\mathrm{mg} \mathrm{dm}^{-3}\right)$ & 80,0 & 63,0 & 1,8 \\
$\mathrm{Ca}^{2+}\left(\mathrm{cmol}_{\mathrm{c}} \mathrm{dm}^{-3}\right)$ & 2,2 & 3,0 & 0,4 \\
$\mathrm{Mg}^{2+}\left(\mathrm{cmol}_{\mathrm{c}} \mathrm{dm}^{-3}\right)$ & 0,9 & 0,8 & 0,2 \\
$\mathrm{Al}^{3+}\left(\mathrm{cmol}_{\mathrm{c}} \mathrm{dm}^{-3}\right)$ & 0,3 & 1,8 & 2,5 \\
$\mathrm{H}+\mathrm{Al}\left(\mathrm{cmol}_{\mathrm{c}} \mathrm{dm}^{-3}\right)$ & 4,5 & 13,0 & 2,3 \\
$\mathrm{SB}\left(\mathrm{cmol}_{\mathrm{c}} \mathrm{dm}^{-3}\right)$ & 3,3 & 4,0 & 2,5 \\
$\mathrm{t}\left(\mathrm{cmol}_{\mathrm{c}} \mathrm{dm}^{-3}\right)$ & 3,6 & 5,7 & 4,8 \\
$\mathrm{~T}\left(\mathrm{cmol}_{\mathrm{c}} \mathrm{dm}^{-3}\right)$ & 7,8 & 17,0 & 47,3 \\
$\mathrm{~V}(\%)$ & 42,1 & 23,2 & 8,0 \\
$\mathrm{~m}(\%)$ & 9,0 & 31,0 & 2,5 \\
$\mathrm{MO}\left(\mathrm{dag} \mathrm{kg}^{-1}\right)$ & 2,7 & 11,1 & 7,2 \\
$\mathrm{P}-\mathrm{rem}\left(\mathrm{mg} \mathrm{L}^{-1}\right)$ & 14,6 & 3,3 & \\
\hline
\end{tabular}


culturais, diferindo apenas na aplicação de nitrogênio. No primeiro experimento forneceu-se $30 \mathrm{~kg} \mathrm{ha}^{-1} \mathrm{de} \mathrm{N}, 64 \mathrm{~kg}$ ha${ }^{1} \mathrm{de}_{2} \mathrm{O}_{5}$ e $48 \mathrm{~kg} \mathrm{ha}^{-1}$ de $\mathrm{K}_{2} 0$ no plantio, e mais $40 \mathrm{~kg} \mathrm{ha}^{-1} \mathrm{de}$ $\mathrm{N}$ em cobertura, a partir do aparecimento da $2^{\mathrm{a}}$ folha trifoliada, tendo como fonte de $\mathrm{N}$ o sulfato de amônio. $\mathrm{O}$ segundo experimento recebeu a mesma adubação com potássio e fósforo, porém não houve aplicação de nitrogênio tanto no plantio, quanto em cobertura. Em ambos os experimentos, utilizou-se o delineamento experimental em blocos casualizados (DBC), com três repetições, sendo as parcelas constituídas por uma linha de três metros de comprimento, espaçadas de $50 \mathrm{~cm}$, contendo 15 sementes por metro linear.

Dessas linhagens foram coletadas de 15 a 20 folhas por parcela no terço médio da planta na floração do feijoeiro, e $100 \mathrm{~g}$ de grãos por parcela após a colheita. As folhas e grãos foram secos em estufa com circulação forçada de ar a $60^{\circ} \mathrm{C}$, até atingir peso constante. A matéria seca das folhas e grãos foi moída em peneira de 40 mesh e o material foi armazenado em frascos de vidro até o momento das determinações químicas.

$\mathrm{O}$ teor de $\mathrm{N}$-total (a) foi determinado pelo método de Kjeldahl, com destilação e titulação feita segundo Tedesco et al. (1995). O N-solúvel (b) foi determinado adicionando-se $30 \mathrm{ml}$ de água destilada à $0,1 \mathrm{~g}$ de folha seca e moída e colocada esta mistura em banho maria à temperatura de $70^{\circ} \mathrm{C}$ durante 45 minutos. Após esfriar à temperatura ambiente, as amostras foram filtradas, obtendose assim um extrato onde foram determinados os compostos nitrogenados solúveis. Em seguida, uma alíquota de $10 \mathrm{ml}$ do extrato filtrado foi colocada em tubo de digestão juntamente com $1,5 \mathrm{~g}$ de $\mathrm{K}_{2} \mathrm{SO}_{4}, 0,3 \mathrm{~g}$ de $\mathrm{CuSO}_{4}$ e $3 \mathrm{ml} \mathrm{de}$ ácido sulfúrico $\left(\mathrm{H}_{2} \mathrm{SO}_{4}\right)$ concentrado. Os processos seguintes: digestão, destilação e titulação foram realizados de forma similar à determinação do N-total. $\mathrm{O} \mathrm{N}-\mathrm{NO}_{3}^{-}$(c) foi determinado de acordo com Cataldo et al. (1975). Os teores de N-orgânico solúvel (aminoácidos, aminas, amidas e proteínas solúveis em água) e o $\mathrm{N}$-insolúvel foram obtidos pela diferença entre (b) e (c) e (a) e (b) respectivamente. As amostras de grãos, por sua vez, foram submetidas, somente, à determinação do N-total, da mesma forma que as amostras de folha.

Os dados de produtividade de grãos, os teores de $(\mathrm{N}$ total na folha e no grão) e as frações do N-total na folha (Nsolúvel, $\mathrm{N}_{-} \mathrm{NO}_{3}^{-} \mathrm{N}$-insolúvel e $\mathrm{N}$-orgânico solúvel) foram submetidos à análise de variância por nível de $\mathrm{N}$ dentro de cada local, às análises de variância conjunta por local e, posteriormente, conjunta de todos os locais. Utilizou-se o programa estatístico SISVAR para realização das análises de variância e testes de comparação de médias (FERREIRA, 2000).
A eficiência do uso de nitrogênio (EUN) foi avaliada pela expressão proposta por Thung (1990). As frações de N foram relacionados a EUN e à produtividade das linhagens. Para auxiliar nesta relação foi realizada a categorização proposta por Fox (1978), sendo as linhagens posicionadas em um gráfico, no qual o eixo "x" é a produção na dose alta e o eixo "y" é a produção na dose baixa. Neste gráfico, quatro quadrantes foram definidos, por retas perpendiculares aos eixos e posicionadas nas médias de produção de cada eixo. As linhagens foram classificadas em função do quadrante que ocuparam. "eficiente responsiva" quando a linhagem produziu acima da média nos dois níveis do nutriente; "ineficiente responsiva" quando a linhagem produziu abaixo da média no nível baixo, mas respondeu ao acréscimo de nutriente, posicionando-se acima da média no nível alto; "eficiente não responsiva" quando a linhagem produziu acima da média no nível baixo, mas não respondeu ao acréscimo de $\mathrm{N}$, ficando abaixo da média no nível alto, e “ineficiente não responsiva" quando a produção da linhagem esteve abaixo da média em ambos os níveis.

\section{RESULTADOS E DISCUSSÃO}

Uma vez que os experimentos com e sem $\mathrm{N}$ foram contíguos em todos os locais, esperava-se que o comportamento das linhagens nos dois experimentos refletisse apenas a diferença no $\mathrm{N}$ aplicado, uma vez que o manejo foi o mesmo, fato que ao que parece realmente ocorreu. Banziger et al. (1997) e Furtini et al. (2006) adotaram esse mesmo procedimento na avaliação de progênies de milho e linhagens de feijoeiro com e sem aplicação de nitrogênio.

As fontes de variação níveis de $\mathrm{N}$, linhagens e locais, bem como a interação níveis $\mathrm{x}$ linhagens apresentaram teste de $\mathrm{F}$ significativo $(\mathrm{P} \leq 0,01)$ para todas as variáveis estudadas.

\section{Produtividade de grãos}

$\mathrm{Na}$ análise de variância conjunta, a estimativa do coeficiente de variação experimental (CV) foi de 24,6\%. Valores semelhantes foram relatados por Furtini et al. (2006) e Matos (2005), em experimentos conduzidos na região, com a cultura do feijoeiro. Considerando-se o tamanho das parcelas e a realização dos experimentos no campo, a precisão é aceitável.

Os locais utilizados diferem em altitude e também quanto à fertilidade do solo. Estas diferenças ambientais explicam a diferença significativa $(\mathrm{P} \leq 0,01)$ entre os locais. As médias de produtividade das linhagens, com aplicação ou não de $\mathrm{N}$, foram maiores em Lavras, seguida por Lambari e por último Ijaci (Tabela 2). Destaca-se que a área, na qual foi realizado o experimento em Lavras, vem sendo cultivada 
há muitos anos e, devido ao uso de adubos e corretivos, a fertilidade do solo era melhor do que nos outros locais (Tabela 1), inclusive, com resíduos de cultura provenientes de cultivos anteriores, refletindo-se certamente em maior disponibilidade de nitrogênio.

As interações significativas $(\mathrm{P} \leq 0,01)$ envolvendo linhagens $\mathrm{x}$ níveis e locais $\mathrm{x}$ linhagens, indicam que $\mathrm{o}$ comportamento das linhagens não foi coincidente nos diferentes níveis e locais. A existência de interação ambientes $\mathrm{x}$ linhagens de feijoeiro é freqüente na região (OLIVEIRA et al., 2006; MATOS et al., 2007). Constatou-se resposta ao N em todos os locais, entretanto, a magnitude de resposta variou entre os locais, sendo mais expressiva em Lavras (Tabela 2).

A produtividade média das linhagens nos ambientes em que houve o fornecimento de nitrogênio (1727 $\mathrm{kg} \mathrm{ha}^{-1}$ ) foi 63,7\% acima da obtida sem aplicação de
N (1055 kg ha-1) (Tabela 3). Resultados de resposta ao nitrogênio na cultura do feijoeiro têm sido freqüentes na literatura (FURTINI et al., 2006; SILVA, 2002; SORATTO et al., 2000). Estes autores verificaram a resposta do feijoeiro à adubação nitrogenada de forma generalizada em diferentes regiões do Brasil, com variações devidas ao manejo do solo, à cultivar, ao clima, à irrigação, época de semeadura e ao sistema de produção.

No ambiente com N, as linhagens ESAL 629 (2212 $\left.\mathrm{kg} \mathrm{ha}^{-1}\right), \mathrm{H}-9$ (2050kg ha-1) e MA-I-2.5 (2022 $\left.\mathrm{kg} \mathrm{ha}^{-1}\right)$ e as linhagens CI-107 (1151 kg ha-1), CNFC 8063 (1324 kg ha'-1) e CNFC 8060 (1535 kg ha $\left.{ }^{-1}\right)$ apresentaram as maiores e as menores produtividades respectivamente. Já no ambiente sem N, a linhagem CNFC 8060, apresentou produtividade média de grãos muito inferior à das demais linhagens que não diferiram entre si (Tabela 3).

Tabela 2 - Produtividade média das linhagens de feijoeiro na ausência e na presença de $\mathrm{N}$ nos diferentes locais de condução dos experimentos.

\begin{tabular}{lcc}
\hline \multirow{2}{*}{ Locais } & \multicolumn{2}{c}{ Produtividade $\left(\mathrm{kg} \mathrm{ha}^{-1}\right)$} \\
\cline { 2 - 3 } & com N & sem N \\
\hline Lavras & 2342 & 1530 \\
Lambari & 1569 & 949 \\
Ijaci & 1275 & 675 \\
\hline
\end{tabular}

Tabela 3 - Produtividade média de grãos das linhagens cultivadas na ausência e na presença de N em Lavras, Lambari e Ijaci, 2005.

\begin{tabular}{|c|c|c|}
\hline \multirow{2}{*}{ Linhagem } & \multicolumn{2}{|c|}{ Produtividade $\left(\mathrm{kg} \mathrm{ha}^{-1}\right)$} \\
\hline & com aplicação de $\mathrm{N}$ & sem aplicação de $\mathrm{N}$ \\
\hline CNFC 8060 & $1535 \mathrm{a}^{1]}$ & $440 \mathrm{~A}$ \\
\hline ESAL 629 & $2212 \mathrm{C}$ & $1199 \mathrm{~B}$ \\
\hline $\mathrm{H}-9$ & $2050 \mathrm{C}$ & $1076 \mathrm{~B}$ \\
\hline CV-46 & $1730 \mathrm{~B}$ & $1049 \mathrm{~B}$ \\
\hline OP-S-30 & $1875 \mathrm{~B}$ & $1148 \mathrm{~B}$ \\
\hline MA I-6.10 & $1724 \mathrm{~B}$ & $1105 \mathrm{~B}$ \\
\hline MA-I-2.5 & $2022 \mathrm{C}$ & $1367 \mathrm{~B}$ \\
\hline RC-I-3 & $1687 \mathrm{~B}$ & $954 \mathrm{~B}$ \\
\hline CIII-R-3-19 & $1784 \mathrm{~B}$ & $1073 \mathrm{~B}$ \\
\hline AN-LAV-51 & $1628 \mathrm{~B}$ & $1070 \mathrm{~B}$ \\
\hline CI-107 & $1151 \mathrm{~A}$ & $885 \mathrm{~B}$ \\
\hline CNFC 8063 & $1324 \mathrm{~A}$ & $1298 \mathrm{~B}$ \\
\hline Médias & $1727 \quad 2]$ & 1055 \\
\hline $\mathrm{CV}$ & & \\
\hline
\end{tabular}

${ }^{11}$ As médias nas colunas seguidas de mesma letra pertencem ao mesmo grupo pelo teste de Scott-Knott $(\mathrm{a}=0,05)$.

${ }^{2]}$ Média com $\mathrm{N}$ foi significativamente diferente da obtida sem $\mathrm{N}$ pelo teste de $\mathrm{F}(\mathrm{a}=0,05)$ 


\section{Frações nitrogenadas}

Os teores de N-total no grão e na folha das linhagens foram mais elevados com aplicação de $\mathrm{N}$ (Tabela 4), corroborando com resultados de muitos trabalhos que têm mostrado o aumento no teor de nitrogênio em diferentes partes da planta de feijoeiro com o aumento da disponibilidade do nutriente (MOLL et al., 1982; PECK \& MACDONALD, 1984; SRIVASTAVA \& ORMOD, 1986).

Os teores de $\mathrm{N}_{-} \mathrm{NO}_{3}{ }_{3}^{-}$na folha foram maiores no ambiente sem N (Tabela 4). Este fato não era esperado, já que, de modo geral, o aumento da disponibilidade de $\mathrm{N}$ acarreta um aumento do teor de nitrato na planta. Guazzelli (1988) em estudos com feijão e Furtini Neto (1988) em trabalho com eucalipto, ambos sob condições de solução nutritiva, verificaram a ocorrência de um incremento no teor de $\mathrm{NO}_{3}^{-}$na folha com aumento da disponibilidade de nitrogênio, embora o teor de $\mathrm{N}_{-} \mathrm{NO}_{3}$ representasse uma fração muito pequena do N-total na folha.

Os teores de N-solúvel, $\mathrm{N}$-insolúvel e N-orgânico solúvel nas folhas de feijoeiro foram maiores com a aplicação de N (Tabela 5). Estes resultados estão de acordo com outros encontrados na literatura, em que se observou que os teores de nitrogênio solúvel aumentaram linearmente, enquanto os de $\mathrm{N}$-insolúvel aumentam somente até certo limite, com o acréscimo da dose de nitrogênio (GUAZZELLI, 1988; MENGEL \& KIRBY, 1982). Como foram aplicados somente dois níveis de $\mathrm{N}$ (0 e $\left.70 \mathrm{~kg} \mathrm{ha}^{-1}\right)$, não foi possível avaliar até que dose o teor de $\mathrm{N}$-insolúvel aumenta com a adição de nitrogênio.

Foram observadas diferenças nos teores de Ntotal na folha e no grão e das frações de $\mathrm{N}$ na folha entre as linhagens estudadas, exceto para o N-solúvel no ambiente com N. Apesar de não ter sido verificada uma relação marcante entre a produtividade das linhagens e as frações de $\mathrm{N}$ na folha, notou-se uma tendência das linhagens ESAL 629 e MA-I-2.5 apresentarem maiores concentrações de N-total no grão e na folha, e de maiores frações de $\mathrm{N}$ nos diferentes locais e níveis de nitrogênio estudados.

\section{Eficiência no uso de nitrogênio}

O comportamento dos índices de EUN das linhagens calculados de acordo com Thung (1990) observados nas safras anteriores (inverno de 2004 e águas 2004/05), não se repetiram no presente trabalho. Além de ocorrer uma diminuição na amplitude dos índices, houve ainda, mudança na classificação das linhagens, possivelmente em função das condições climáticas, do cultivo em áreas com solos diferentes. Também a diferença na quantidade de $\mathrm{N}$ aplicada, pode ter contribuído para a não repetibilidade da EUN das linhagens.

De maneira geral não houve relação entre a EUN e a produtividade das linhagens com os teores das frações de $\mathrm{N}$ nas folhas e do $\mathrm{N}$-total no grão e na folha. Assim sendo, o metabolismo do N, avaliado apenas pelo fracionamento do nitrogênio, proposto no presente trabalho, parece não ter sido adequado para explicar as diferenças de produtividade e da eficiência do uso de $\mathrm{N}$ entre as linhagens estudadas. Há que se considerar que a complexa dinâmica do $\mathrm{N}$ em experimentos no campo, possa ter contribuído para esses resultados. Guazzelli (1988) em estudos de linhagens de feijoeiro submetidas a diferentes doses de nitrato em solução nutritiva, também, não verificou relação entre a produção de biomassa e EUN com as frações de $\mathrm{N}$ na parte aérea do feijoeiro.

Nas tabelas 4 e 5 pode-se observar que as linhagens ESAL 629, MA-I-2.5 e H-9, que tiveram produtividades e índices de EUN constantes em todos os locais estudados, apresentaram tendência a terem maiores concentrações de $\mathrm{N}$-total nos grãos e nas folhas e maiores frações de $\mathrm{N}$ na folha tanto no ambiente com e sem a aplicação do nutriente. A tabela 6 ilustra a relação entre as frações de N, do N-total nos grãos e nas folhas com a produtividade e com a EUN das linhagens, utilizando-se a categorização sugerida por Fox (1978).

Embora o comportamento das linhagens não tenha se mantido constante nas categorizações realizadas para a produtividade, teores de $\mathrm{N}$-total nas folhas e nos grãos e das frações nitrogenadas nas folhas, observa-se que as linhagens ESAL 629 e MA-I-2.5 apresentaram comportamento mais constante em relação a estas variáveis, exceto para o teor de $\mathrm{N}$-total nos grãos, comportando-se como eficientes e responsivas (E/R). Essa observação confirma que estas linhagens certamente apresentam uma maior estabilidade em relação à produtividade e à EUN.

Um aspecto que deveria ser considerado em futuros trabalhos seria a análise de outros componentes do metabolismo do nitrogênio, tais como as enzimas redutase do nitrato (RN), glutamina sintetase (GS), glutamato sintase (GOGAT) e nitrogenase, visando complementar os estudo das frações nitrogenadas, na tentativa de explicar o comportamento diferencial das linhagens quanto a EUN. 
Frações nitrogenadas e eficiência nutricional...

Tabela 4 - Teores médios de nitrogênio total na folha (NTf) e no grão (NTg) e o nitrato na folha $\left(\mathrm{N}_{-} \mathrm{NO}_{3}^{-}\right)$das linhagens avaliadas na ausência e na presença de $\mathrm{N}$ em Lavras, Lambari e Ijaci.

\begin{tabular}{|c|c|c|c|c|c|c|}
\hline \multirow{2}{*}{ Linhagem } & \multicolumn{2}{|c|}{$\operatorname{NTf}\left(\mathrm{mg} \mathrm{g}^{-1}\right)$} & \multicolumn{2}{|c|}{$\operatorname{NTg}\left(\mathrm{mg} \mathrm{g}^{-1}\right)$} & \multicolumn{2}{|c|}{$\mathrm{N}-\mathrm{NO}_{3}^{-}\left(\mathrm{mg} \mathrm{g}^{-1}\right)$} \\
\hline & $\operatorname{com} \mathrm{N}$ & $\operatorname{sem} \mathrm{N}$ & $\operatorname{com} \mathrm{N}$ & sem N & $\operatorname{com} \mathrm{N}$ & sem N \\
\hline CNFC 8060 & $41,2 b^{1]}$ & $35,2 \mathrm{~b}$ & $37,3 \mathrm{~b}$ & $31,1 \quad \mathrm{~A}$ & $1,04 \mathrm{c}$ & $1,18 \mathrm{c}$ \\
\hline ESAL 629 & 41,2 B & $37,4 \mathrm{c}$ & 34,0 a & 34,4 B & $1,12 \mathrm{c}$ & $1,09 \mathrm{~b}$ \\
\hline $\mathrm{H}-9$ & $41,0 \quad \mathrm{~B}$ & $38,0 \quad \mathrm{c}$ & $38,3 \mathrm{~b}$ & $38,2 \mathrm{C}$ & $1,10 \mathrm{c}$ & $1,19 \mathrm{c}$ \\
\hline CV-46 & $40,7 \quad \mathrm{~B}$ & 31,0 a & $37,8 \mathrm{~b}$ & $36,7 \quad \mathrm{C}$ & 0,85 a & $1,07 \mathrm{~b}$ \\
\hline OP-S-30 & 38,9 A & $34,3 \mathrm{~b}$ & 35,6 a & $33,7 \quad \mathrm{~B}$ & $1,05 \mathrm{c}$ & $1,17 \mathrm{c}$ \\
\hline MA I-6.10 & $39,4 \quad \mathrm{~A}$ & $34,0 \mathrm{~b}$ & 35,3 a & $34,3 \quad \mathrm{~B}$ & $1,09 \mathrm{c}$ & $1,14 \mathrm{c}$ \\
\hline MA-I-2.5 & $40,1 \mathrm{~A}$ & $35,3 \mathrm{~b}$ & 35,2 a & $34,8 \quad \mathrm{~B}$ & $1,09 \mathrm{c}$ & $1,18 \mathrm{c}$ \\
\hline RC-I-3 & $39,2 \mathrm{~A}$ & $36,4 \mathrm{c}$ & $40,2 \mathrm{c}$ & $39,2 \mathrm{C}$ & $0,94 \mathrm{~b}$ & $1,04 \mathrm{~b}$ \\
\hline CIII-R-3-19 & 42,9 B & $33,4 \quad b$ & $37,6 \mathrm{~b}$ & 33,7 B & $1,05 \mathrm{c}$ & $1,06 \mathrm{~b}$ \\
\hline AN-LAV-51 & $39,7 \mathrm{~A}$ & $35,1 \quad b$ & $38,4 \quad b$ & $35,7 \quad \mathrm{~B}$ & 0,88 a & 0,96 a \\
\hline CI-107 & $38,2 \mathrm{~A}$ & 30,3 a & $40,7 \mathrm{c}$ & $38,3 \mathrm{C}$ & $1,21 \mathrm{~d}$ & $1,16 \mathrm{c}$ \\
\hline CNFC 8063 & $40,6 \quad \mathrm{~B}$ & $35,9 \mathrm{~b}$ & 35,9 a & $35,3 \quad \mathrm{~B}$ & $0,91 \mathrm{~b}$ & $1,06 \mathrm{~b}$ \\
\hline Médias & $40,2^{2]}$ & 34,7 & 37,2 & 35,4 & 1,03 & 1,11 \\
\hline $\mathrm{CV}$ & 6,1 & & 5 , & & & \\
\hline
\end{tabular}

${ }^{1]}$ As médias nas colunas seguidas de mesma letra pertencem ao mesmo grupo pelo teste de Scott-Knott $(\alpha=0,05)$.

${ }^{2]}$ Média com $N$ foi sign ificativamente diferente da obtida sem $N$ pelo teste de $F(\alpha=0.05)$.

Tabela 5 - Teores médios de nitrogênio solúvel (NS), insolúvel (NI) e orgânico solúvel (NOS) das linhagens avaliadas na ausência e na presença de $\mathrm{N}$ em Lavras, Lambari e Ijaci.

\begin{tabular}{|c|c|c|c|c|c|c|}
\hline \multirow{2}{*}{ Linhagem } & \multicolumn{2}{|c|}{$\mathrm{NS}\left(\mathrm{mg} \mathrm{g}^{-1}\right)$} & \multicolumn{2}{|c|}{$\mathrm{NI}\left(\mathrm{MG} \mathrm{g}^{-1}\right)$} & \multicolumn{2}{|c|}{$\operatorname{NOS}\left(\mathrm{mg} \mathrm{g}^{-1}\right)$} \\
\hline & $\operatorname{com} \mathrm{N}$ & $\operatorname{sem} \mathrm{N}$ & $\operatorname{com} \mathrm{N}$ & $\operatorname{sem} \mathrm{N}$ & $\operatorname{com} \mathrm{N}$ & sem N \\
\hline CNFC 8060 & $6,4 a^{1]}$ & $5,6 \mathrm{c}$ & $34,8 \quad \mathrm{~b}$ & $29,6 \mathrm{c}$ & $5,4 \mathrm{~b}$ & 4,4 \\
\hline ESAL 629 & 6,7 a & $5,9 \mathrm{c}$ & $34,5 \mathrm{~b}$ & $31,5 \mathrm{~d}$ & $5,6 \mathrm{~b}$ & 4,8 \\
\hline H-9 & 5,8 a & $5,6 \mathrm{c}$ & $35,2 \mathrm{~b}$ & $32,4 \mathrm{~d}$ & 4,7 a & 4,4 \\
\hline CV-46 & 6,3 a & $5,1 \mathrm{~b}$ & $34,6 \mathrm{~b}$ & 25,9 a & $5,4 \mathrm{~b}$ & $4,1 \quad \mathrm{~b}$ \\
\hline OP-S-30 & 6,4 a & $5,4 \mathrm{~b}$ & 32,5 a & $28,9 \mathrm{c}$ & $5,3 \mathrm{~b}$ & $4,2 \mathrm{~b}$ \\
\hline MA I-6.10 & 6,4 a & $5,2 \mathrm{~b}$ & 33,0 a & $28,8 \mathrm{c}$ & $5,4 \mathrm{~b}$ & $4,1 \mathrm{~b}$ \\
\hline MA-I-2.5 & 6,4 a & $5,9 \mathrm{c}$ & 33,7 a & $29,5 \mathrm{c}$ & $5,3 \mathrm{~b}$ & 4,7 c \\
\hline RC-I-3 & 6,4 a & $6,1 \mathrm{c}$ & 32,9 a & 30,4 c & $5,4 \mathrm{~b}$ & 5,0 \\
\hline CIII-R-3-19 & 6,3 a & $5,5 \mathrm{c}$ & $36,6 \mathrm{~b}$ & $27,9 \mathrm{~b}$ & $5,3 \mathrm{~b}$ & 4,4 c \\
\hline AN-LAV-51 & 6,5 a & $5,4 \mathrm{~b}$ & 33,1 a & 29,7 c & $5,6 \mathrm{~b}$ & 4,4 \\
\hline CI-107 & 6,1 a & 4,7 a & 32,1 a & 25,6 a & 4,9 a & 3,5 a \\
\hline CNFC 8063 & $6,5 \mathrm{a}$ & $5,7 \mathrm{c}$ & $34,0 \quad b$ & $30,2 \mathrm{c}$ & $5,6 \mathrm{~b}$ & 4,6 \\
\hline Médias & $6,3^{2]}$ & 5,5 & 33,9 & 29,7 & 5,3 & 4,4 \\
\hline $\mathrm{CV}$ & \multicolumn{2}{|c|}{8,8} & \multicolumn{2}{|c|}{6,8} & \multicolumn{2}{|c|}{10,6} \\
\hline
\end{tabular}

${ }^{1]}$ As médias nas colu nas seguidas de mesma letra pertencem ao mesmo grupo pelo teste de Scott-Knott $(\alpha=0,05)$.

${ }^{2]}$ Média com $\mathrm{N}$ foi sign ificativamente diferente da obtida sem $\mathrm{N}$ pelo teste de $\mathrm{F}(\alpha=0.05)$. 
Tabela 6- Classificação das linhagens quanto à eficiência de utilização de nitrogênio.

\begin{tabular}{lccccccc}
\hline \multicolumn{1}{c}{ Linhagens } & $\begin{array}{c}\text { Produtividade } \\
\left(\mathrm{kg} \mathrm{ha}^{-1}\right)\end{array}$ & $\begin{array}{c}\mathrm{NTf} \\
\left(\mathrm{mg} \mathrm{g}^{-1}\right)\end{array}$ & $\begin{array}{c}\mathrm{NTg} \\
\left(\mathrm{mg} \mathrm{g}^{-1}\right)\end{array}$ & $\begin{array}{c}\mathrm{NS} \\
\left(\mathrm{mg} \mathrm{g}^{-1}\right)\end{array}$ & $\begin{array}{c}\mathrm{N}-\mathrm{NO}_{3}^{-} \\
\left(\mathrm{mg} \mathrm{g}^{-1}\right)\end{array}$ & $\begin{array}{c}\mathrm{NI} \\
\left(\mathrm{mg} \mathrm{g}^{-1}\right)\end{array}$ & $\begin{array}{c}\mathrm{NOS} \\
\left(\mathrm{mg} \mathrm{g}^{-1}\right)\end{array}$ \\
\hline CNFC 8060 & $\mathrm{I} / \mathrm{NR}$ & $\mathrm{E} / \mathrm{R}$ & $\mathrm{I} / \mathrm{R}$ & $\mathrm{E} / \mathrm{R}$ & $\mathrm{E} / \mathrm{R}$ & $\mathrm{E} / \mathrm{R}$ & $\mathrm{XE} / \mathrm{R}$ \\
ESAL 629 & $\mathrm{E} / \mathrm{R}$ & $\mathrm{E} / \mathrm{R}$ & $\mathrm{I} / \mathrm{NR}$ & $\mathrm{E} / \mathrm{R}$ & $\mathrm{I} / \mathrm{R}$ & $\mathrm{E} / \mathrm{R}$ & $\mathrm{E} / \mathrm{R}$ \\
$\mathrm{H}-9$ & $\mathrm{E} / \mathrm{R}$ & $\mathrm{E} / \mathrm{R}$ & $\mathrm{E} / \mathrm{R}$ & $\mathrm{E} / \mathrm{NR}$ & $\mathrm{E} / \mathrm{R}$ & $\mathrm{E} / \mathrm{R}$ & $\mathrm{XE} / \mathrm{NR}$ \\
RC-I-3 & $\mathrm{I} / \mathrm{NR}$ & $\mathrm{E} / \mathrm{NR}$ & $\mathrm{E} / \mathrm{R}$ & $\mathrm{E} / \mathrm{R}$ & $\mathrm{I} / \mathrm{NR}$ & $\mathrm{E} / \mathrm{NR}$ & $\mathrm{E} / \mathrm{R}$ \\
OP-S-30 & $\mathrm{E} / \mathrm{R}$ & $\mathrm{I} / \mathrm{NR}$ & $\mathrm{I} / \mathrm{NR}$ & $\mathrm{I} / \mathrm{R}$ & $\mathrm{E} / \mathrm{R}$ & $\mathrm{I} / \mathrm{NR}$ & $\mathrm{I} / \mathrm{XR}$ \\
CIII-R-3-19 & $\mathrm{E} / \mathrm{R}$ & $\mathrm{I} / \mathrm{R}$ & $\mathrm{I} / \mathrm{R}$ & $\mathrm{XE} / \mathrm{XR}$ & $\mathrm{I} / \mathrm{R}$ & $\mathrm{I} / \mathrm{R}$ & $\mathrm{XE} / \mathrm{XR}$ \\
CV-46 & $\mathrm{I} / \mathrm{R}$ & $\mathrm{I} / \mathrm{R}$ & $\mathrm{E} / \mathrm{R}$ & $\mathrm{I} / \mathrm{XR}$ & $\mathrm{I} / \mathrm{NR}$ & $\mathrm{I} / \mathrm{R}$ & $\mathrm{I} / \mathrm{R}$ \\
MA-I-2.5 & $\mathrm{E} / \mathrm{R}$ & $\mathrm{E} / \mathrm{NR}$ & $\mathrm{I} / \mathrm{NR}$ & $\mathrm{E} / \mathrm{R}$ & $\mathrm{E} / \mathrm{R}$ & $\mathrm{E} / \mathrm{NR}$ & $\mathrm{E} / \mathrm{XR}$ \\
MA I-6.10 & $\mathrm{E} / \mathrm{NR}$ & $\mathrm{I} / \mathrm{NR}$ & $\mathrm{I} / \mathrm{NR}$ & $\mathrm{I} / \mathrm{R}$ & $\mathrm{E} / \mathrm{R}$ & $\mathrm{I} / \mathrm{NR}$ & $\mathrm{I} / \mathrm{R}$ \\
AN-LAV-51 & $\mathrm{E} / \mathrm{NR}$ & $\mathrm{E} / \mathrm{NR}$ & $\mathrm{E} / \mathrm{R}$ & $\mathrm{I} / \mathrm{R}$ & $\mathrm{I} / \mathrm{NR}$ & $\mathrm{E} / \mathrm{NR}$ & $\mathrm{XE} / \mathrm{R}$ \\
CI-107 & $\mathrm{I} / \mathrm{NR}$ & $\mathrm{I} / \mathrm{NR}$ & $\mathrm{E} / \mathrm{R}$ & $\mathrm{I} / \mathrm{NR}$ & $\mathrm{E} / \mathrm{R}$ & $\mathrm{I} / \mathrm{NR}$ & $\mathrm{I} / \mathrm{NR}$ \\
CNFC 8063 & $\mathrm{E} / \mathrm{NR}$ & $\mathrm{E} / \mathrm{R}$ & $\mathrm{I} / \mathrm{NR}$ & $\mathrm{E} / \mathrm{R}$ & $\mathrm{I} / \mathrm{NR}$ & $\mathrm{E} / \mathrm{R}$ & $\mathrm{E} / \mathrm{R}$ \\
\hline
\end{tabular}

E - eficiente; I - ineficiente, $\mathrm{R}$ - responsiva e NR - não responsiva e X quando está exatamente no limite entre eficiente e não eficiente e entre responsiva e não responsiva.

\section{CONCLUSÕES}

A produtividade de grãos com $\mathrm{N}$ foi $63,7 \%$ superior à obtida sem $\mathrm{N}$.

As linhagens de feijoeiro diferiram com relação a EUN, sendo as mais eficientes as linhagens ESAL 629 e MA-I-2.5. Estas linhagens também apresentaram as maiores produtividades de grãos em todos os locais e níveis de N.

Os teores de nitrato foram maiores sem a aplicação de $\mathrm{N}$, por outro lado, os teores das demais frações de $\mathrm{N} \mathrm{e}$ do N-total na folha e no grão foram mais elevados quando houve o fornecimento de nitrogênio.

Não se constatou boa associação entre os teores de N-total e frações de $\mathrm{N}$ nas folhas (N-nítrico, $\mathrm{N}$-insolúvel, $\mathrm{N}$-solúvel e N-orgânico solúvel) com a EUN entre as linhagens estudadas. Contudo, nas linhagens ESAL $629 \mathrm{e}$ MA-I-2.5 observou-se indicações que as frações de $\mathrm{N}$ e $\mathrm{N}$-total na folha poderia explicar a maior EUN

\section{REFERÊNCIAS BIBLIOGRÁFICAS}

ALVES, V. G. Resposta do feijoeiro a doses de nitrogênio no plantio e cobertura e à inoculação de sementes com rizóbio. 2002. 46 p. Dissertação (Mestrado em Fitotecnia) - Universidade Federal de Lavras, Lavras, 2002.

BANZIGER, M. et al. Efficiency of high-nitrogen selection environments for improving maize for lownitrogen target environments. Crop Science, Madson, v. 37, n. 4, p. 1103-1109, 1997.
BRASIL, E. C.; ALVES, V. M. C.; MARRIEL, I. E.; PITTA, G. V.E.; CARVALHO, J. G. DE. Matéria seca e acúmulo de nutrientes em genótipos de milho contrastantes quanto a aquisição de fósforo. Ciência e Agrotecnologia, Lavras, v. 31, n. 3, p. 704-712, maio/jun., 2007.

CATALDO, D. A.; HAARON, M.; SCHRADER, L. E.; YOUNGS, V. L. Rapid colorimetric determination of nitrate in plant tissue of nitration of salicylic acid.

Communications in Soil Science and Plant Analysis, New York, v. 6, p. 71-90, 1975.

FERREIRA, D. F. Análises estatísticas por meio do Sisvar para Windows versão 4.0. In: REUNIÃO ANUAL DA REGIÃO BRASILEIRA DA SOCIEDADE INTERNACIONAL DE BIOMETRIA, 45., 2000, São Carlos, SP. Anais... São Carlos: UFSCar, 2000. p. 255-258.

FOX, R. H. Selection for phosphorus efficiency in corn. Comunications in Soil Science and Plant Analysis, New York, v. 9, p. 13 -27, 1978.

FRANCO, A. A. Nutricional restraints for tropical grain legume symbiosis. In: VICENT, J. M. K.; WHITNEY, J. (Eds.). Exploiting the legume-Rhizobium in tropical agriculture. Hawai: University of Hawai, 1997. p. 237-252.

FURTINI, I. V.; RAMALHO, M. A. P.; ABREU, A. F. B.; FURTINI NETO, A. E. Resposta diferencial de linhagens de feijoeiro ao nitrogênio. Ciência Rural, Santa Maria, v. 36, n. 6, p. 1696-1700, 2006. 
FURTINI NETO, A. E.Efeito do enxofre no crescimento e assimilação de nitrogênio por diferentes espécies de eucalipto. 1988. 95 f. Dissertação (Mestrado em Solos e Nutrição de Plantas) - Universidade Federal de Lavras, Lavras, 1988.

GUAZZELLI, E. M. F. M. Efeito de nitrato e amônio no crescimento, assimilação e eficiência de utilização do nitrogênio por cultivares de feijoeiro (Phaseolus vulgaris $\mathbf{L}$.) na fase inicial de crescimento. 1988. $112 \mathrm{f}$. Dissertação (Mestrado em Solos e Nutrição de Plantas) Universidade Federal de Lavras, Lavras, 1988.

MATOS, J. W. de. Análise crítica do programa de melhoramento genético do feijoeiro da UFLA no período de 1974 a 2004. 2005. Tese (Doutorado em Genética e Melhoramento de Plantas) - Universidade Federal de Lavras, Lavras, 2005.

MATOS, J. W. DE.; RAMALHO, M. A. P.; ABREU, Â. DE F. B. Trinta e dois anos do programa de melhoramento genético do feijoeiro comum em Minas Gerais. Ciência e Agrotecnologia, Lavras, v. 31, n. 6, p. 1749-1754, nov./dez., 2007.

MENGEL, K.; KIRKBY, E. A. Principles of plant nutrition. Berna: International Potash Institute, 1982. 655 p.

MOLL, R. H.; KAMPRATH, E. J.; JACKSON, W. A. Analysis and interpretation of factors which contribute to eficiency of nitrogen utilization. Agronomy Journal, Madison, v. 74, p. 562-564, 1982.

OLIVEIRA, G. V.; CARNEIRO, P. C. S.; CARNEIRO, J. E. S.; CRUZ, C. D. Adaptabilidade e estabilidade de linhagens de feijão comum em Minas Gerais. Pesquisa Agropecuária Brasileira, Brasília, v. 41, n. 2, p. 257-265, 2006.

PECK, N. H.; MacDONALD, G. E. Snap bean plant responses to nitrogen fertilization. Agronomy Journal, Madison, v. 76, p. 247-253, Mar./Apr. 1984.

SILVA, F. C. Eficiência de uso de nitrogênio por seis cultivares de milho. 2002. 61 f. Dissertação (Mestrado em Agronomia) - Universidade Estadual Paulista, Ilha Solteira, 2002.

SILVA, M. V. da. Aplicação foliar de cobalto, inoculação com rizóbio e adubação nitrogenada na cultura do feijoeiro. 2002. 54 p. Dissertação (Mestrado em Fitotecnia) - Universidade Federal de Lavras, Lavras, 2002.

SORATTO, R. P.; SILVA, T. R. B.; CHIDI, S. N.; ARF, O.; BUZETTI, S. Feijoeiro irrigado e a aplicação de nitrogênio em cobertura e molibdênio via foliar. Cultura Agronômica, Viçosa, v. 9, p. 115-132, 2000.

SRIVASTAVA, H. S.; ORMOND, D. P. Effects of nitrogen dioxide and nitrate nutrition on nodulation nitrogenase activity, growth and nitrogen content of bean plants. Plant Physiology, Maryland, v. 81, n. 3, p. 737-741, July 1986.

TEDESCO, M. J.; GIANELLO, C.; BISSANI, C. A.; BOHNEN, H.; VOLKWEISS, S. J. Análises de solo, plantas e de outros materiais. Porto Alegre: UFRGS, 1995. 174 p. (Boletim técnico, 5).

THUNG, M. Phosporus: a limiting nutrient in bean (Phaseolus vulgaris L.) production in Latin America and field screening for efficiency and response. In: BASSAM et al. (Eds.). Genetic aspects of plant mineral nutrition. Dordrecht: Netherlands Kluwer, 1990. p. 501521.

VIEIRA, C. Adubação mineral e calagem. In: VIEIRA, C.; PAULA JUNIOR, T. J.; BORÉM, A. (Eds.). Feijão: aspectos gerais e cultura no Estado de Minas. Viçosa: UFV, 1998. p. 123-152.

VIEIRA, N. M. B. Crescimento e marcha de absorção de nutrientes no feijoeiro cvs. BRS-MG Talismã e Ouro Negro, em plantio direto e convencional. 2006. 145 p. Dissertação (Mestrado em Fitotecnia) - Universidade Federal de Lavras, Lavras, 2006. 